\title{
Design of multi node CAN bus in high frequency system
}

\author{
Zhang Lei ${ }^{1}$ \\ ${ }^{1}$ (713Research Institute of CSSC, Henan Zhengzhou, 450015)
}

\begin{abstract}
In order to ensure the reliability of multiple nodes CAN bus in high frequency system, we need to find the design method of CAN bus. In this paper, we rely on the test platform to carry out tests and summarize the design method of elimination high frequency system.
\end{abstract}

\section{Introduction}

Controller Area Network(CAN) is a bus protocol which has been widely used in various industrial fields. Compared with other networked control systems, the bus system can realize data transmission between nodes in distributed control system, more real-time and reliable. But in high frequency systems, due to the introduction of high frequency interference, it will lead to the serious decline of bus transmission quality. Therefore, we need to find a solution to high frequency interference, and verify its effectiveness.

In this paper, building a high frequency system test platform. The main methods to solve the high frequency interference are tested. It provides a basis for improving the reliability of multiple nodes CAN bus in high frequency system.

\section{High frequency electromagnetic interference}

Electromagnetic interference needs three conditions: Interference source, Receiving unit, Coupling channe.

1) Interference source

Interference source mainly has the following kinds:(1)

Transient voltage wave caused by load mutation in AC power grid. (2) High frequency oscillation voltage is produced in the process of strong current components passing through the current. (3) During the switching process of GTR, large pulse current causes magnetic or electromagnetic interference.(4)High frequency radiation of DC servo motor due to the change of current direction.

2) Receiving unit

Receiving unit is the component that vulnerable to high frequency interference, and causing voltage distortion. CAN bus due to the transmission of $5 \mathrm{~V}$ voltage, the voltage amplitude is small, vulnerable to interference.

3) Coupling channel

The interference signal can be coupled to the sensitive element in many ways. Delivery methods include:
Conduction coupling, Common impedance coupling, and Radiation electromagnetic field coupling.

(1) Conduction coupling: It is the transmission of unwanted electromagnetic energy through a conductor. They propagate interference through power lines, signal lines and control lines. Wires and conductors are important ways to transmit interference.

(2) Common impedance coupling: In PWM systems, there is a common reference point for signal transmission-Potential reference point, this creates a common impedance, the current flowing into the common impedance couples the interference to other circuits. Poor grounding mode is the main cause of common impedance coupling.

(3) Radiation electromagnetic field coupling: It refers to the magnetic flux produced by the current flowing in the conductor, and the induced voltage generated by the coupling of adjacent wires or circuits through mutual inductance. The main ways of inductive coupling are transformer coupling and parallel wire coupling.

\section{Introduction of test platform}

In order to better reflect the high frequency environment, the test platform consists of two $30 \mathrm{Kw}$ drivers, four sets of $3 \mathrm{Kw}$ drivers, they are respectively installed in two power cabinets. In order to maximize the deterioration of the installation environment, install one $30 \mathrm{~kW}$ and three $3 \mathrm{~kW}$ drives in power cabinet 2 , other drives are installed in power cabinet 1 . The controller is installed in the control box. The cable link diagram between cabinets is shown in figure 1. 


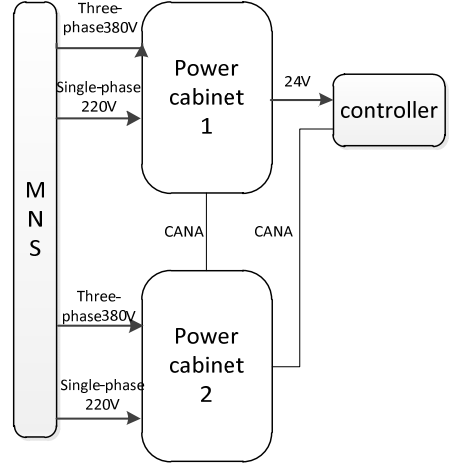

Figure 1 Cable connection diagram between cabinets

The system CAN bus topology is shown in Figure 2 . There are seven nodes on the bus, the communication lines between nodes are connected in the form of end to end connection, connect terminal resistor to power cabinet 1 and control box respectively, the bus resistance is $60 \mathrm{ohm}$, meet the requirements of CAN bus.

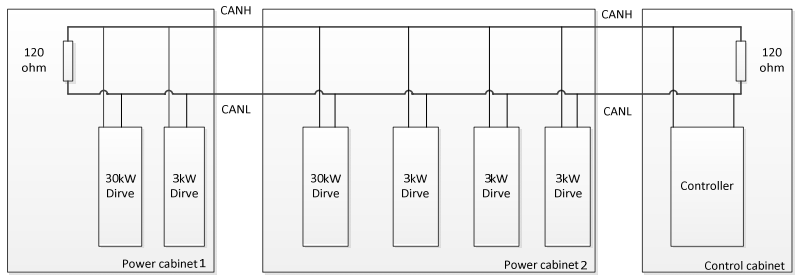

Figure 2 CAN bus topology diagram

\section{Experimental verification}

AC servo driver is used in the test platform, in laboratory environment, power supply of municipal power grid, the total power of the driver is $72 \mathrm{~kW}$, it will not cause sudden change of power grid. Drive independent power supply, as shown in Figure 3.Therefore, the main interference source of the test platform is the interference generated by GTR in the driver at the moment of switching.

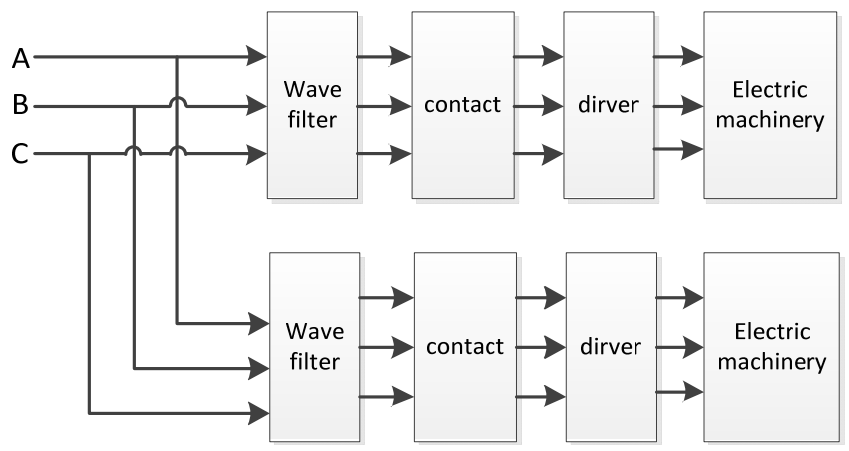

Figure 3 Power cabinet internal cable diagram

The platform mainly tests the interference transmission path, including driver grounding, cable inside and outside the cabinet, component layout etc.

(1) Influence of component arrangement on interference

In order to test the influence of component arrangement on interference introduction, the two power

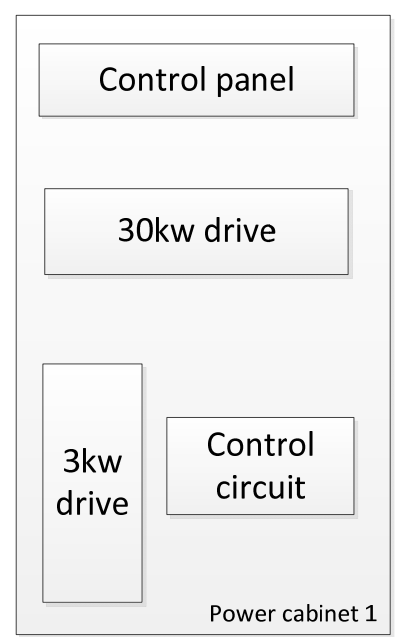

cabinets are arranged in different ways. As shown in Figure 4, the control circuit in power cabinet 2 is next to the control panel, clear division with power line. In power cabinet 1, the control circuit is separated from the control panel by $30 \mathrm{~kW}$ power line, it's easier to introduce interference.

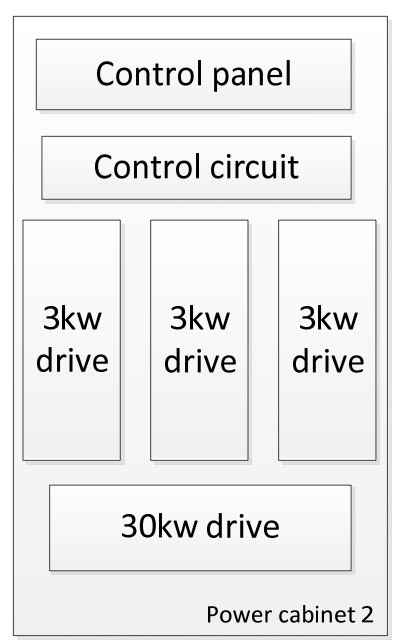

Figure 4 Configuration of power cabinet components

Start power cabinet 1 and power cabinet 2 respectively, observe the bus waveform, as shown in Figure 5. 

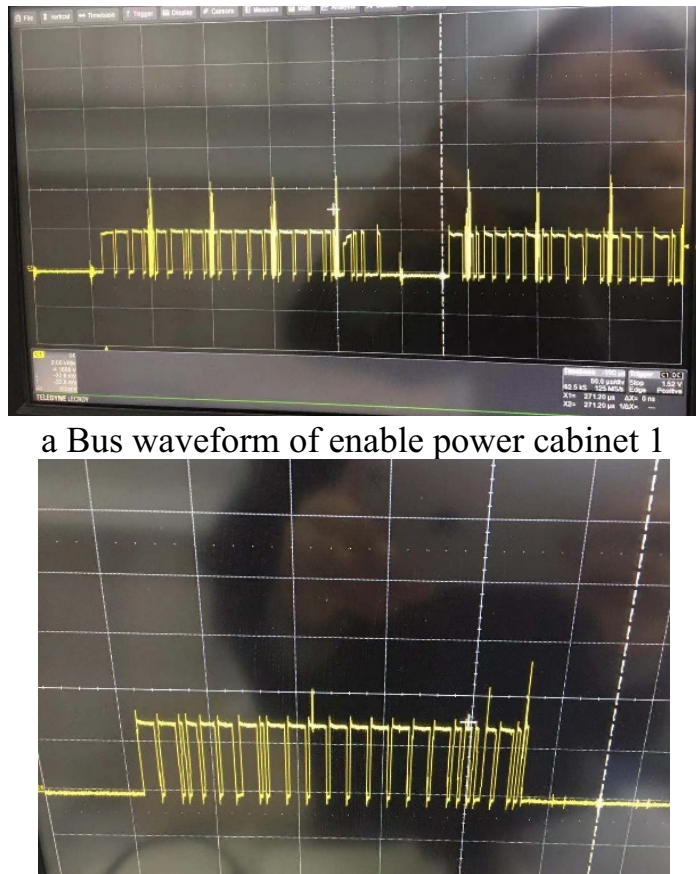

b Bus waveform of enable power cabinet 2

Figure 5 Influence test of component installation position

Less drives in power cabinet 1, the installation conditions are relatively loose, however, due to the cross of control cable and power cable, the driver can obviously interfere with CAN bus. Through comparison, it can be seen that to eliminate high-frequency interference, it is necessary to plan the installation of components in the cabinet, and the cross of control cable and power cable may lead to the introduction of interference.

(2) Influence of driver grounding on interference

The grounding mode of drive in power cabinet 2 is changed from simultaneous grounding of input and output to grounding of input end. After enabling, the bus waveform is shown in Figure 6. Compared with $b$ in Figure 5,it can be seen that the interference of single ended grounding bus of driver is obviously strengthened, therefore, the input and output terminals of the driver are grounded at the same time to achieve the complete grounding of the equipment.。

(3) Influence of cable laying on interference

Do not change the installation position of components in power cabinet 1 , separate the control circuit from the power circuit, shielded and single end grounded. CAN cable away from power cable, The waveform of CAN bus is shown in Figure 7.

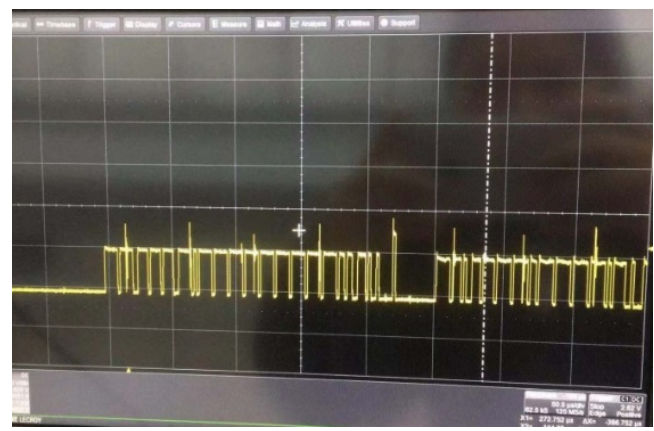

Figure 6 Waveform of drivers input grounding cabinet 1

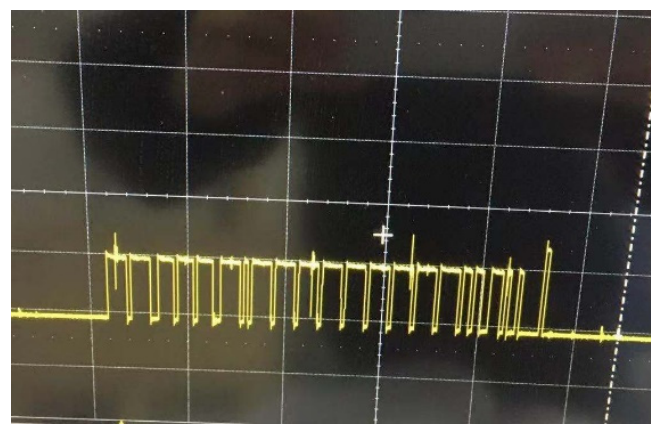

Figure 7 Change the laying waveform in cabinet 2 
Compare with a in Figure 5, it can be seen that the interference can be obviously improved by adding shielding measures.

\section{Test conclusion}

Through the test, it can be seen that the main improvement measures of high frequency interference include:

(1) The input and output terminals of the driver are grounded at the same time.

(2) The power line and control line are partitioned, avoid crossing each other and parallel cable routing.

(3) The cable in the cabinet is shielded and grounded at one end.

(4) Keep communication cables away from power cables.

\section{Reference}

1. Meng linzhi Analysis and design of electromagnetic compatibility Vol. 39 No. 5 Oct. 2019

2. WANG Kai, FENG Xin bo Research on Anti-EMI Technology of Equipments in Communication Engineering Vol.3 No.8 Aug. 2019

3. Shen xuemei research on intelligent processing and parameter modeling of EMI conductive noise journal of NanJing normal university 2006.12

4. Cao haiyang Study on Electromagnetic Compatibility of High Power Variable Frequency Speed-Regulating System doctor's thesis of China Mining university 2019 page7-12

5. Chi baichao exploring the EMC performance design and analysis method of CAN bus communication electronic technology and software engineering. 